\title{
Large Bleeding Tonsillar Mass in a Child - Rare Presentation of Non-Hodgkin Lymphoma
}

\section{Ramesh Bhat Y and Anand Kumar Patil}

Department of Paediatrics, Kasturba Medical College, Manipal, Manipal Academy of Higher Education (MAHE) University, Karnataka, India

\section{Correspondence:}

Ramesh Bhat Y

Department of Paediatrics,

Kasturba Medical College,

Manipal, Manipal Academy of Higher Education (MAHE) University,

Karnataka, India

Email: docrameshbhat@yahoo.co.in

DOI: $10.3126 /$ jnps.v40i3.30821

Submitted on: 2020-06-24

Accepted on: 2020-07-03

Acknowledgements: None

Funding: Nil

Conflict of Interest: None declared

Permission from IRB: Yes
To cite this article: Bhat RY, Patil AK Large bleeding tonsillar mass in a child - rare presentation of non-hodgkin lymphoma. J Nepal Paediatr Soc. 2020;40(3):270-3

\begin{abstract}
Tonsillar malignancy is the underlying etiology of only a very small percentage of paediatric unilateral tonsillar enlargements. Further, because of rarity of tonsillar malignancy in children, the diagnosis may be delayed. Authors describe a large bleeding tonsillar mass in a child who remained undiagnosed for two months but finally diagnosed with non-Hodgkin lymphoma at the institution.
\end{abstract}

Key words: bleeding; child; lymphoma; tonsil mass

This work is licensed under creative common attribution 3.0 license 


\section{INTRODUCTION}

Unilateral tonsillar enlargement (UTE) is uncommon in children. Malignancy in UTE is rare. ${ }^{1-4}$ Primary malignant tumours of the tonsil constitute about $12 \%$ of oral cavity neoplasms, squamous cell carcinoma accounts for $85 \%$ to $90 \%$ of tonsillar malignancies, and the remaining $10 \%$ to $15 \%$ are lymphomas and other rarer tumours. ${ }^{1}$ The literature regarding paediatric tonsil cancer is limited. The case reports in paediatric patients describe tonsillar enlargement over a time of days to weeks who were eventually diagnosed with malignant UTE. Authors describe a UTE in a 13 year old boy who remained undiagnosed for two months with multiple health care visits.

\section{CASE REPORT}

A 13 year old boy presented with swelling in the throat since two months and intermittent bleeding since seven days. He received multiple courses of antibiotics without relief of his symptoms. Several physician contacts failed to make any concrete diagnosis. The swelling gradually progressed; he had difficulty in swallowing, pain while chewing, and developed snoring. There was no history of restriction of movements of mouth, earache, ear discharge, recurrent cold and cough, fever or weight loss. He was seen at cancer hospital few days ago where biopsy of the lesion was performed. The biopsy showed predominantly degenerated cells with few atypical lymphoid cells. Bone marrow study showed scattered lymphoid cells. As there was no concrete diagnosis, he was discharged. The symptoms worsened further and he approached our institute again.

Physical examination revealed height of $106 \mathrm{~cm}$ and weight of $14.8 \mathrm{~kg}$. A swelling was present on the right side of the neck which was firm in consistency. Throat examination with tongue depressor showed solitary mass, approximately 5 $\mathrm{cm} \times 5 \mathrm{~cm}$ in the right tonsillar fossa (Figure 1). His vital signs, rest of the general physical and systemic examination were normal.

Complete blood count revealed haemoglobin of $12.3 \mathrm{~g} / \mathrm{dL}$; total leucocyte count $6.1 \times 10^{3} / \mu \mathrm{L}$ with lymphocytes: $53 \%$ and neutrophils $29 \%$; platelet count $1.81 \times 10^{3} / \mu \mathrm{L}$. Peripheral smear did not show abnormal cells. Tests for HIV, hepatitis B and C viruses were negative. Serum electrolytes, blood glucose, uric acid and creatinine were normal. Lactate dehydrogenase was $547 \mathrm{U} / \mathrm{L}$.

The biopsy of the mass showed altered architecture, diffuse infiltration by a monotonous population of medium to large cells with round nuclei, fine chromatin, one to three nucleoli, scant basophilic cytoplasm and brisk mitotic activity. A diagnosis of non-Hodgkin's lymphoma (NHL) was considered. Bone marrow study revealed infiltration by NHL. CD20 and CD10 positivity and, CD3 and Tdt negative on immunohistochemistry (IHC) suggested Burkitt lymphoma (BL). CSF study was normal. He was started on intravenous antibiotics and fluids along with other symptomatic management. After the diagnosis of malignancy, the child was transferred to paediatric oncology department. An appropriate chemotherapy regimen was initiated. He was relieved of symptoms by the end of one month and he is presently asymptomatic under regular follow up.

\section{DISCUSSION}

Tonsillar malignancy is rare in children. ${ }^{1-3,5}$ UTE may be a sign of potential malignancy. Primary malignant tumours of the tonsil include squamous cell carcinoma ( $85 \%$ to $90 \%$ ), lymphoma ( $10 \%$ to $15 \%$ ) and others. Tonsillar lymphoma may present as an UTE. ${ }^{4}$ The discrepancy of tonsillar size may be largely attributed to differences in tonsil position relative to the depth of the tonsillar fossa. ${ }^{6}$ If the degree of asymmetry corresponds with a difference in score on the Brodsky scale of $\geq 2$, then it suggests true asymmetry. In the present case there was a large unilateral tonsillar mass which had a tendency to bleed following food intake. Secondary

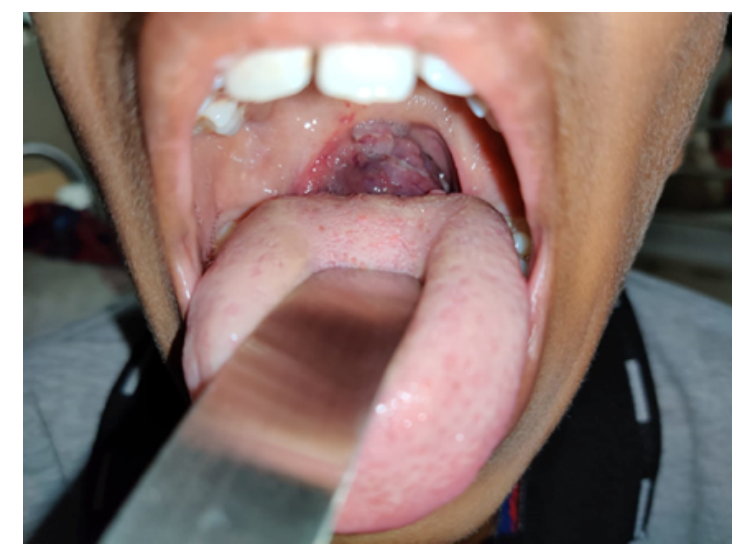

Figure 1: Large right tonsillar mass 
malignancy of the tonsil is very rare. A study involving 138 paediatric tonsillar malignancies in USA reported NHL in $84.1 \%{ }^{5}$ Burkitt lymphoma constituted $31.1 \%$ among them.

The literature regarding paediatric tonsil cancer is limited given its rarity. The tonsil enlargement may be initially considered benign in children. ${ }^{4}$ Therefore diagnosis of tonsil cancer may be delayed for days to weeks. Rapid tonsillar enlargement over three to 12 weeks usually suggests malignancy. ${ }^{6}$ In the present case, the child remained undiagnosed for about two months. Even the biopsy did not give any concrete evidence of malignancy initially. Concurrent cervical lymphadenopathy increases the likelihood of malignancy. ${ }^{4-6}$ The present case did not have cervical lymphadenopathy. Male predominance in paediatric tonsillar malignancy was reported by Adil et al. $^{5}$ A systematic review of tonsillar lymphoma in children found tonsillar asymmetry being the common symptom. ${ }^{7}$ Additional symptoms include lymphadenopathy in children of six to 10 years of age, snoring in less than five years of age and dysphagia among children between 11 to 18 years of age. Fever and weight loss are not significant findings in any age group.

In the present case, IHC suggested aggressive subtype - BL. The peak incidence of BL in children occurs at age 11 years. BL in children belongs to three clinical forms: endemic, sporadic and immunodeficiency-associated. ${ }^{1,4} \mathrm{BL}$ is commoner in male gender. In the present case, HIV screening was negative. The case appeared to be of sporadic type. Sporadic BL is rare, highly aggressive and usually has an abdominal presentation, often with massive disease. Generalised lymphadenopathy is common. Jaw or facial involvement, bone marrow and CNS involvement occurs in $25 \%, 30 \%$ and $15 \%$ respectively at the time of initial presentation. ${ }^{1}$
In the present case, malignancy was limited to tonsil. BL tumour cells are monomorphic, mediumsized cells with round nuclei, multiple nucleoli, and basophilic cytoplasm. They express CD 19, CD 20, CD 22, CD 79a and CD 10 and lack CD 5, BCL - 2, TdT, and CD 23. The immunohistochemistry of the present case showed these features.

Further, misleading presentations of paediatric tonsillar malignancies are not uncommon. Symptoms similar to peritonsillar infection in a four year old boy was reported by Fabian et al. ${ }^{8}$. The biopsy confirmed BL and chemotherapy resulted in cure with no recurrence in him. Same authors reported a tonsillar malignancy mimicking peritonsillar abscess in an eight year old boy. Prompt treatment of paediatric tonsillar cancer may lead to survival rate of $>90 \% .{ }^{8,9}$

\section{CONCLUSIONS}

Massive unilateral tonsillar enlargement in children may suggest malignancy. As the majority of paediatric tonsillar malignancies are aggressive non-Hodgkin lymphoma subtypes, they need timely diagnosis and effective therapy for better outcome.

\section{REFERENCES}

1. Boerma EG, van Imhoff GW, Appel IM, Veeger NJ, Kluin PM, Kluin-Nelemans JC. Gender and age-related differences in Burkitt lymphoma-epidemiological and clinical data from The Netherlands. Eur J Cancer. 2004; 40(18):2781-7. DOI:https://doi.org/10.1016/j.ejca.2004.09.004

2. Blum KA, Lozanski G, Byrd JC. Adult Burkitt leukemia and lymphoma. Blood. 2004;104(10):3009-20. DOI: 10.1182/blood-2004-02-0405. 
3. Muwakkit SA, Razzouk BI, Shabb NS, Hancock ML, Dabbous I, Firzli S, et al. Clinical Presentation and Treatment Outcome of Children With Burkitt Lymphoma in Lebanon: A Single Institution's Experience. J Pediatr Hematol Oncol. 2004;26(11):749-53. DOI: 10.1097/00043426-200411000-00012

4. Cunha KCCMS, Oliveira MCLA, Gomes ACS, de Castro LPF, Viana MB. Clinical Course and Prognostic Factors of Children With Burkitt's Lymphoma in a Developing Country: The Experience of a Single Centre in Brazil. Rev Bras Hematol Hemoter. 2012;34(5):361-6. DOI: 10.5581/1516-8484.20120093

5. Adil EA, Medina G, Cunningham MJ. Pediatric Tonsil Cancer: A National and Institutional Perspective. J Pediatr 2018;197:255-61. DOI: 10.1016/j.jpeds.2018.01.022

6. Harley EH. Asymmetric tonsil size in children. Arch Otolaryngol Head Neck Surg. 2002;128:767-9. DOI: 10.1001/ archotol.128.7.767

7. de Carvalho GM, Pauna HF, Crespo AN, Gusmão RJ, Guimarães AC. Tonsillar Lymphoma in Children According to Age Group: A Systematic Review. Iran J Otorhinolaryngol. 2018;30(97): 69-75. PMID: 29594072

8. Fabian D, Mahida JB, Pluto CP, Thompson BP, Minneci PC, Deans KJ et al. Pediatric Tonsillar Malignancies: Misleading Presentations. Inter J Otorhinolaryngol. 2015;2(1):4.

9. Janza TA, Nagasubramaniana R, Wei JL. A Survival Analysis of Pediatric Tonsillar Malignancies and Review of a Rare Case. Int J Pediatr Otorhinolaryngol. 2019;118:160-4. DOI: https://doi.org/10.1016/j.ijporl.2018.12.033 